\title{
BMJ Open Health outcomes in international migrant children: protocol for a systematic review
}

\author{
Alice Jane Armitage (D), Michelle Heys, Irina Lut (D), Pia Hardelid
}

To cite: Armitage AJ, Heys M, Lut I, et al. Health outcomes in international migrant children: protocol for a systematic review. BMJ Open 2021;11:e041173. doi:10.1136/ bmjopen-2020-041173

- Prepublication history and additional supplemental material for this paper are available online. To view these files, please visit the journal online (http://dx.doi.org/10.1136/ bmjopen-2020-041173)

Received 01 June 2020 Revised 02 March 2021 Accepted 03 March 2021
Check for updates

(C) Author(s) (or their employer(s)) 2021. Re-use permitted under CC BY-NC. No commercial re-use. See rights and permissions. Published by BMJ.

UCL Great Ormond Street Institute of Child Health, University College London, London, UK

Correspondence to Dr Alice Jane Armitage; alice.armitage.13@ucl.ac.uk

\section{ABSTRACT}

Introduction Migration status is a key determinant of health, but health outcomes among migrant children and young people (CYP), that is, those aged under 18 years, are poorly understood. A 'healthy migrant' effect has been demonstrated among adults, but evidence for the same effect in CYP is lacking. No large studies or reviews exist reporting comprehensive or holistic health outcomes among migrant CYP. We aim to identify and synthesise original quantitative research on health of migrant CYP to explore the relations between migration status and health outcomes.

Methods and analysis A search of PubMed/Medline, Embase, Cochrane and grey literature sites will be undertaken for any original quantitative research on health outcomes of migrant CYP from 01 January 2000 onwards. Outcomes addressed: mortality, communicable diseases, non-communicable diseases, nutritional status, mental health, disability, vaccine coverage, and accidental and non-accidental injuries (including assault and abuse). Search results will be screened and presented in a Preferred Reporting Items for Systematic Reviews and Meta-Analyses flow diagram.

The Newcastle-0ttawa Scale assessment tool will be used to assess study quality. If feasible, depending on study availability data heterogeneity (explored using $\mathrm{I}^{2}$ statistic), results will be pooled for meta-analysis. If sufficient data are available, a priori defined subgroup analyses will be undertaken. A narrative quantitative synthesis will be presented, taking account of study quality and assessed risk of bias.

The anticipated search completion date is 01 June 2021 with write-up completed by 01 April 2022.

Ethics and dissemination Formal ethical approval will not be sought as we will be accessing data already in the public domain. This review will be submitted for publication in a high-impact journal and presented at international conferences. The results of this work will be shared with groups of migrant children as part of an ongoing engagement project.

PROSPERO registration number CRD42020166305.

\section{INTRODUCTION}

Migration status is known to be a key determinant of health. ${ }^{1}$ There is no international consensus on the definition of migrant; here we use the term to describe international migrants, as suggested by the United Nations

\section{Strengths and limitations of this study}

This protocol is written in line with Preferred Reporting Items for Systematic Review and MetaAnalysis Protocols reporting guidelines for systematic review protocols.

- This systematic review will address a clear research gap on health outcomes of migrant children and young people.

- Well-established systematic review methodology will allow for evidence-based recommendations for policy around migrant children based on available data and identification of key gaps in the research evidence.

- Review conclusions are likely to be limited by the quality and quantity of available studies.

definition: 'someone who changes his or her country of usual residence, irrespective of the reason for migration or legal status'. ${ }^{2}$ This includes refugees and asylum seekers, as well as economic migrants and international students. ${ }^{2}$ Adult migrants may be young and healthy, and a 'healthy migrant' effect, whereby migrants have better health status than the population of the host country, has been demonstrated. ${ }^{3}$

Migrant populations may experience poverty, social inequality or persecution at their destination, which could compound physical and mental health burdens associated with country of origin, reasons for displacement and circumstances of their journey. Children and young people (CYP: those under the age of 18 years) are further impacted by the health of their caregivers, and by their inherent physical and social vulnerabilities, particularly to malnutrition, communicable diseases, disrupted education, violence and exploitation. ${ }^{4-7}$ Unlike adults migrating for work or education, who are likely to be healthy, CYP are significantly less likely to be the drivers of their own migration. There may also be families who migrate to seek healthcare for CYP with chronic conditions. Therefore, it is not yet known whether 
there will be the same 'healthy migrant' effect in CYP as observed in adult migrants.

Many high-income countries place restrictions on migrant CYP's entitlement to health services. ${ }^{8}$ Unmet health needs in CYP are known to be associated with poor adult health. ${ }^{9}$ However, health needs and associated health outcomes among migrant CYP are poorly understood $^{10}$ and infrequently reported in the literature, preventing a population-based approach to planning health services. This is in part due to poor quality and quantity of data on this topic. Routinely collected healthcare datasets rarely include migration status for children. Most studies on the health of migrants only include children as a subgroup, if included at all. There is a lack of data on migrant health spanning larger geographical regions and crossing borders, barriers to data linkage, and an associated lack of large scale studies or reviews. ${ }^{11}$ The lack of healthcare data on migrant CYP has been identified as an unmet research need, ${ }^{11}$ as well as a rights of the child issue. ${ }^{12}$

A recent systematic review on mortality of international migrants provided evidence that, on average, international migrants have lower mortality than the host population. ${ }^{3}$ However, mortality from specific causes, such as violence and infectious diseases, was higher among migrants. ${ }^{3}$ This systematic review also highlighted a lack of data on the health of vulnerable migrant groups such as asylum seekers, refugees and undocumented migrants, ${ }^{3}$ and authors cautioned against generalising results to these groups. Similarly, the majority of studies reflect migration into high-income countries with a noted evidence gap for data on migrants, particularly refugees, in low-income and middle-income countries. Health outcomes for migrants from different geographical regions and countries are likely to be related to the socioeconomic conditions of the origin and host countries. For example, a study of perceived well-being in adolescent migrants living in Canada showed different rates of health complaints based on country and region of origin. ${ }^{13}$

Other comprehensive systematic review evidence addresses perinatal outcomes among migrants, indicating increased maternal mortality, preterm birth and congenital abnormalities, as well as barriers to access and use of healthcare. ${ }^{14}$ Systematic review evidence from 2018 demonstrates that migrant children use healthcare services less than non-migrant populations, with the exception of emergency services, although their rate of hospital admission is higher. ${ }^{15}$

As mortality is a relatively rare outcome in childhood, wider health outcomes are required to reflect the impact of migration on health. Over the last decade, the life course approach to health has been adopted by multiple international organisations, such as the $\mathrm{WHO},{ }^{16}$ and is reflected in approaches such as the 'survive and thrive' strategy of the sustainable development goals. ${ }^{17}$ Rather than seeing health outcomes as discrete and unrelated, this approach takes a comprehensive and holistic view of health. It allows for early influences on risk factors for long-term conditions potentially presenting later in life and the biopsychosocial model of child and adolescent health to be taken into account. ${ }^{16}$ There have been no comprehensive systematic reviews on a range of migrant child health outcomes across the life course either internationally or in the UK. Following an International Prospective Register for Systematic Reviews (PROSPERO) search, no similar review is planned at present.

The proposed systematic review will therefore address a clear evidence gap. We have designed this review to address comprehensive health outcomes across the life course of CYP (ie, up to age 18 years), in addition to mortality.

\section{AIMS AND OBJECTIVES}

The aim of the systematic review will be to summarise the available evidence base regarding a range of key health outcomes of migrant CYP across the childhood life course.

Our specific objectives are to:

1. Identify global original quantitative research on health outcomes for migrant CYP, and to compare this with CYP in the host population where data are available. Where there is no control group or the control group are another migrant group, the studies will be included in the quantitative narrative synthesis.

2. Undertake meta-analyses of specific health outcomes if the data allow, for example, mortality, vaccination coverage (see eight domains listed below). Similarly, if sufficient data are available, subgroup analyses, decided a priori, will include breakdown of health outcomes by:

- Age group (1-4, 5-9, 10-17 years).

- Migrant subgroup (refugee, asylum seeker, child of economic migrants, student).

- Migrant country of origin and destination (according World Bank national income groups). ${ }^{18}$

- Study quality as assessed by Newcastle-Ottawa Scale (NOS) tool $^{19}$ (see below).

\section{METHODS AND ANALYSIS}

This protocol is written with reference to the Preferred Reporting Items for Systematic Review and Meta-Analysis Protocols reporting guidelines for systematic review protocols. $^{20}$

\section{Patient and public involvement}

No patient involved.

\section{Eligibility}

This systematic review will include published studies presenting original data on health outcomes of migrant CYP, that is, those living in a different country from that of their birth, including observational studies (cohort and case-control studies, and cross-sectional surveys), systematic review and randomised controlled trials. Studies in any setting and from any country will be included. 
For the purposes of this review, we are including studies pertaining to CYP who are international migrants, i.e. living in a country other than that of their birth, irrespective of the birth place of their parents. Therefore, only studies on first-generation migrant children will be included, that is, we would not include studies on children born to parents who were originally migrants. In view of this, and in view of existing systematic review evidence, studies will be excluded that pertain exclusively to maternal and/or perinatal outcomes as these will not address the outcomes for CYP who have themselves migrated. The perinatal period in this systematic review is defined as birth up until the child's first birthday unless it is explicitly stated that the child migrated during this period. If studies specifically pertain to infants under 1 year who have migrated then these data will be included and analysed within the 1-4 age group. We will exclude studies that only include patients from intensive care or high dependency settings and where health outcomes do not fall within defined areas (see below). In view of existing systematic review evidence and the defined outcomes areas, we will not include studies exclusively reporting hospital attendance or admission rates without other health outcomes presented. We will also exclude research letters, studies where the abstract or full text is not available, and studies where it is not possible to obtain an English translation. Restricting systematic reviews to English language publications is routine practice and has been shown not to significantly affect results regarding empirical studies. ${ }^{21}$

\section{Outcomes of interest}

The outcomes will be grouped into the following eight areas, chosen to represent key health outcomes across the childhood life course. Outcomes are chosen with reference to the Global Burden of Disease Study $2017^{22}$ and to reflect the 'survive and thrive' strategy of the sustainable development goals. ${ }^{17}$ More emphasis has been placed on health outcomes where quantitative data may be available, where definitions are recognised internationally and where the outcomes are plausibly affected my migration status. For each outcome, a finite list of more common conditions has been chosen.

\section{Mortality (by age group $1-4,5-9,10-17$ years)}

If data are available following identification of studies, mortality will be broken down by age group and compared with the host population. In view of perinatal studies being excluded and focus being on children who have themselves migrated, we are excluding infant mortality (deaths under the age of 1 year).

\section{Communicable diseases (incidence/prevalence)}

Systematic review evidence suggests that despite the 'healthy migrant' effect, rates of infectious diseases are higher among migrant populations. ${ }^{3}$ The search strategy will focus on HIV, hepatitis B, tuberculosis (active and latent), sexually transmitted diseases (chlamydia and gonorrhoea), schistosomiasis and parasitic infections. ${ }^{3}$

\section{Non-communicable diseases}

With reference to the global data, the search strategy will focus on neoplasms, asthma and other chronic respiratory conditions, epilepsy and type 1 diabetes mellitus. With chronic conditions characterised by occasional exacerbations, such as asthma, the focus will be on exacerbations of the condition as opposed to baseline prevalence.

\section{Overnutrition and undernutrition}

Forced migration of children may be associated with periods of food insecurity both before and during migration, with associated morbidity. ${ }^{23}$ Following migration to middle-income and high-income countries, migrant children are at risk of becoming overweight or obese. ${ }^{24}$ We are therefore seeking to identify studies addressing both undernutrition and overnutrition in migrant children. The search strategy will focus on terms around malnutrition, undernutrition, underweight, low body mass index (BMI), high BMI, overweight and obesity. Micronutrient deficiencies, such as vitamin deficiencies, requiring blood tests to identify, are considered outside the scope of this review.

\section{Mental health outcomes}

Poor mental health is increasingly recognised as an unmet health need in childhood and adolescence and being identified as prevalent among migrant populations. ${ }^{10} 25$ The search strategy will focus on post-traumatic stress disorder, psychosis, depression, self-harm and suicide.

\section{Disability}

Disability may be higher among migrant children from countries with poor health infrastructure and has been identified as a significant unmet health need among migrant children. ${ }^{2326}$ The search strategy will focus on hearing impairment, deafness, visual impairment, blindness, cerebral palsy, autism, learning difficulties and/or developmental delay.

\section{Vaccine coverage and uptake}

Lack of access to preventative healthcare and disruption to healthcare access in migrant CYP affect vaccination coverage. Following migration, catch-up immunisation programmes depend on timely and coordinated healthcare input. The search strategy will focus on immunisation, vaccination and specific vaccine-preventable pathogen targets (polio, diphtheria, pertussis, measles, mumps, rubella, hepatitis B) combined with vaccinespecific terms (vaccination, immunisation, immunity).

\section{Accidental and non-accidental injuries (eg, assault and abuse)}

Road traffic accidents and interpersonal violence are examples of accidental injuries that may be associated with migration. It is also known that migrant CYP are at increased risk of assault and abuse both historically (in their country of origin and during transit) and following 
migration. ${ }^{23} 27$ Rates of sexual assault and abuse are also high, particularly among forced migrants, and will be included in this category. The search strategy will focus on road traffic accidents or injuries, interpersonal or domestic violence, physical or sexual assault or abuse, sexual violence and rape.

\section{Search strategy}

The electronic databases PubMed/Medline, Embase and Cochrane will be searched with date range from 01 January 2000 onwards. A grey literature search will also be undertaken including the following websites: Organisation for Economic Co-operation and Development, WHO Global Health Observatory, Health Evidence Network, Health for Undocumented Migrants and Asylum seekers Network and the International Organization of Migration. We will also undertake reference checking for selected manuscripts and search conference proceedings from international conferences relevant to migrant child health.

The search strategy will use keywords and index terms around migrant status, CYP and the eight areas of health outcomes as described above. A draft search strategy for Ovid Medline for Mortality is attached (online supplemental appendix 1). The finalised search strategy for all outcomes will be published online and any amendments identified (PROSPERO register).

\section{Selection process}

Search results will be exported to EPPI V.4 software ${ }^{28}$ for screening and selection. Two independent reviewers (AJA and IL) will screen all titles and abstracts. Full manuscripts will be screened when it is not clear from the title or abstract whether the study meets the inclusion criteria. Where there is disagreement between the two reviewers, the study will be escalated to a third reviewer ( $\mathrm{MH}$ or $\mathrm{PH}$ ) to resolve. Following the screening of full text, articles will be assessed for eligibility; a Preferred Reporting Items for Systematic Reviews and Meta-Analyses (PRISMA) flow diagram will be produced and the PRISMA checklist followed. ${ }^{29}$

\section{Data synthesis and analysis}

Data will be extracted and entered into a Microsoft Excel spreadsheet by a single reviewer (AJA or IL). We will extract the following data items: demographic features (age, sex and country/countries of origin of CYP), study design, country/countries of arrival (study setting), study period, study population, presence of control or comparator group, outcomes presented (using predefined categories as listed above), outcome measures (rate ratio, HR or OR), follow-up period and funding source.

The NOS $^{19}$ assessment tool will be used to assess the quality of studies. The NOS assigns a 'star system' across three domains: the selection of the study groups; the comparability of the groups; and the ascertainment of either the exposure or outcome of interest for casecontrol or cohort studies, respectively. The NOS score for each study will be presented. A sensitivity analysis will be undertaken by rerunning the meta-analyses excluding any low-quality outliers on the NOS.

The decision to meta-analyse study results will depend on the availability of studies pertaining to the various outcomes and heterogeneity of the data presented. The $\mathrm{I}^{2}$ statistic will be used to explore heterogeneity of studies. A small number of studies (fewer than three) or $\mathrm{I}^{2}$ of $>75 \%$ will be adopted as the threshold for decision not to undertake meta-analysis. $^{30}$

Studies presenting original data on one or more of the eight defined health outcomes will be considered for inclusion in meta-analyses. Summary parameters for most outcomes are likely to be rate ratio, HR or OR. If appropriate, other measures such as prevalence or vaccine uptake rates, will be used. Study results will be pooled for meta-analysis using STATA V.14 using a random effects model (Der-Simonian and Laird method) ${ }^{31}$ and presented in forest plots. The likelihood of publication bias will be explored using funnel plots if enough studies are identified. We acknowledge that if a small number of studies is identified, it may not be possible to assess publication bias.

If sufficient data are available, the following subgroup analyses will be undertaken: breakdown of health outcomes by age group ( $1-4,5-9,10-17$ years $)$, by migrant subgroup (refugee, asylum seeker, child of economic migrants, student), by migrant destination (World Bank income group ${ }^{18}$ ) and by study quality as assessed by the NOS tool. ${ }^{19}$

A quantitative narrative synthesis will be undertaken of studies that are not included in the meta-analysis, guided by the Systematic review Without Meta-analysis guidelines. ${ }^{32}$ We will clearly set out why studies are not included in a meta-analysis; the diversity of studies will be addressed (including populations, methodology and outcomes) and the completeness of outcome data. Studies will be grouped for synthesis according to the eight predefined outcomes (table 1). Any quantitative effect sizes presented (that have not been amenable to meta-analysis) will be presented in tables. Statistics will not be combined for presentation outside of the meta-analysis. Where the heterogeneity cannot be explored using the $\mathrm{I}^{2}$ statistic, heterogeneity will be informally explored by ordering studies according to characteristics including outcomes and population. Studies will be prioritised based on assessed risk of bias, sample and effect size and relevance to the research question. For each outcome, a description of synthesised findings will be made including certainty of results (with reference to $\mathrm{p}$ values and CIs where available), conclusions will take account of quality of included studies and the assessed risk of bias.

Bias due to confounding must be considered when addressing migration as a risk factor for health outcomes: migration is inevitably correlated with race/ ethnicity, poverty and educational level. Bias due to missing data, selection bias and reporting bias will also be considered. 
Table 1 Research question in PICOS format

1. Population, or participants Children and young people (CYP), defined as those under the age of 18 years and conditions of interest

$\begin{aligned} & \text { 2. Interventions or exposures } \\ & \text { 3. Comparisons or control } \\ & \text { groups }\end{aligned}$ CYP who have not migrated, described as 'the host population'

4. Outcomes of interest
$\begin{aligned} & \text { 1. Mortality (age group: } 1-4,5-9,10-17 \text { years), infants are excluded unless clearly stated } \\ & \text { that they have migrated after birth, in which case they will be included in } 1-4 \text { age group } \\ & \text { 2. Communicable diseases (incidence/prevalence) } \\ & \text { 3. Non-communicable diseases } \\ & \text { 4. Overnutrition and undernutrition } \\ & \text { 5. Mental health outcomes } \\ & \text { 6. Disability } \\ & \text { 7. Vaccine coverage } \\ & \text { 8. Accidental and non-accidental injuries (eg, assault and abuse) }\end{aligned}$

5. Setting

6. Study designs
Studies in any setting and from any country will be included

All studies presenting original data, including observational (cohort, case-control and crosssectional studies), systematic reviews and randomised controlled trials reporting quantitative data on health outcomes in international migrant CYP
Anticipated timeline for review: searches completion on 01 June 2021, screening of search results by 01 December 2021, data analysis and write-up by 01 April 2022.

\section{Ethics and dissemination}

Formal ethical approval will not be sought in line with systematic review guidelines: we will only be accessing data already in the public domain. This review will make up part of an MD(Res) thesis. We will submit results for publication in a high-impact peer-reviewed journal. Results will be presented at international conferences in the areas of migrant health and paediatrics and disseminated to policymakers via the Children and Families Policy Research unit at UCL. The results of this review will be relevant for UK and international stakeholders in the area of migrant child health. Funding has been secured for a separate study involving unaccompanied asylum-seeking children in the UK, a particularly vulnerable group of migrant children. The results of this work will be shared with this group as part of an ongoing engagement project.

This protocol has been submitted to PROSPERO and revised based on their recommendations. PROSPERO registration number CRD42020166305.

\section{Twitter Alice Jane Armitage @ArmitageAlice}

Contributors The protocol was conceived by all authors, written by AJA and reviewed by $\mathrm{PH}, \mathrm{MH}$ and IL prior to submission. $\mathrm{PH}$ is the guarantor of the review.

Funding The authors have not declared a specific grant for this research from any funding agency in the public, commercial or not-for-profit sectors.

Competing interests None declared.

Patient consent for publication Not required.

Provenance and peer review Not commissioned; externally peer reviewed.

Supplemental material This content has been supplied by the author(s). It has not been vetted by BMJ Publishing Group Limited (BMJ) and may not have been peer-reviewed. Any opinions or recommendations discussed are solely those of the author(s) and are not endorsed by BMJ. BMJ disclaims all liability and responsibility arising from any reliance placed on the content. Where the content includes any translated material, BMJ does not warrant the accuracy and reliability of the translations (including but not limited to local regulations, clinical guidelines, terminology, drug names and drug dosages), and is not responsible for any error and/or omissions arising from translation and adaptation or otherwise.

Open access This is an open access article distributed in accordance with the Creative Commons Attribution Non Commercial (CC BY-NC 4.0) license, which permits others to distribute, remix, adapt, build upon this work non-commercially, and license their derivative works on different terms, provided the original work is properly cited, appropriate credit is given, any changes made indicated, and the use is non-commercial. See: http://creativecommons.org/licenses/by-nc/4.0/.

\section{ORCID iDs}

Alice Jane Armitage http://orcid.org/0000-0001-6972-3651

Irina Lut http://orcid.org/0000-0002-9075-648X

Pia Hardelid http://orcid.org/0000-0002-0154-1306

\section{REFERENCES}

1 Abubakar I, Aldridge RW, Devakumar D, et al. The UCL-Lancet Commission on migration and health: the health of a world on the move. The Lancet 2018;392:2606-54.

2 Perruchoud R. Persons falling under the mandate of the International organization for migration (IOM) and to whom the organization may provide migration services. Int J Refug Law 1992;4:205-15.

3 Aldridge RW, Nellums LB, Bartlett S, et al. Global patterns of mortality in international migrants: a systematic review and metaanalysis. Lancet 2018;392:2553-66.

4 Manhica H, Almquist Y, Rostila M, et al. The use of psychiatric services by young adults who came to Sweden as teenage refugees: a national cohort study. Epidemiol Psychiatr Sci 2017;26:526-34.

5 Buchanan A, Kallinikaki T. Meeting the needs of unaccompanied children in Greece. Int Soc Work 2018:0020872818798007.

6 Chavez L, Menjívar C. Children without borders: a mapping of the literature on unaccompanied migrant children to the United States. Migraciones Internacionales 2017;5:71-111.

7 Kloning T, Nowotny T, Alberer M, et al. Morbidity profile and sociodemographic characteristics of unaccompanied refugee minors seen by paediatric practices between October 2014 and February 2016 in Bavaria, Germany. BMC Public Health 2018;18:983.

8 Stubbe Østergaard L, Norredam M, Mock-Munoz de Luna C, et al. Restricted health care entitlements for child migrants in Europe and Australia. Eur J Public Health 2017;27:869-73.

9 Hargreaves DS, Elliott MN, Viner RM, et al. Unmet health care need in US adolescents and adult health outcomes. Pediatrics 2015;136:513-20. 
10 Carrasco-Sanz A, Leiva-Gea I, Martin-Alvarez L, et al. Migrant children's health problems, care needs, and inequalities: European primary care paediatricians' perspective. Child Care Health Dev 2018;44:183-7.

11 Kerbl R, Grois N, Popow C, et al. Pediatric healthcare for refugee minors in Europe: steps for better insight and appropriate treatment. J Pediatr 2018;197:323-4.

12 Carballo M, Hargreaves S, Gudumac I, et al. Evolving migrant crisis in Europe: implications for health systems. Lancet Glob Health 2017;5:e252-3.

13 Borraccino A, Charrier L, Berchialla P, et al. Perceived well-being in adolescent immigrants: it matters where they come from. Int $\mathrm{J}$ Public Health 2018;63:1037-45.

14 Heslehurst N, Brown H, Pemu A, et al. Perinatal health outcomes and care among asylum seekers and refugees: a systematic review of systematic reviews. BMC Med 2018;16:89.

15 Markkula N, Cabieses B, Lehti V, et al. Use of health services among international migrant children - a systematic review. Global Health 2018;14:52.

16 Jacob C, Baird J, Barker M. The importance of a life course approach to health: chronic disease risk from preconception through adolescence and adulthood. Geneva: WHO, 2017.

17 Temmerman M, Khosla R, Bhutta ZA, et al. Towards a new global strategy for women's, children's and adolescents' health. BMJ 2015;351:h4414.

18 Bank W. New country classifications by income level: 2018-2019. [TheDATABlog] 2018.

19 Wells G. The Newcastle-Ottawa scale (NOS) for assessing the quality of non randomised studies in meta-analyses, 2001. Available: http://www ohri ca/programs/clinical_epidemiology/ oxford

20 Moher D, Shamseer L, Clarke M, et al. Preferred reporting items for systematic review and meta-analysis protocols (PRISMA-P) 2015 statement. Syst Rev 2015;4:1.

21 Morrison A, Polisena J, Husereau D, et al. The effect of Englishlanguage restriction on systematic review-based meta-analyses: a systematic review of empirical studies. Int J Technol Assess Health Care 2012;28:138-44.

22 Roth GA, Abate D, Abate KH, et al. Global, regional, and national age-sex-specific mortality for 282 causes of death in 195 countries and territories, 1980-2017: a systematic analysis for the global burden of disease study 2017. The Lancet 2018;392:1736-88.

23 Hjern A. Health of refugee and migrant children: technical guidance 2018.

24 Labree LJW, van de Mheen $\mathrm{H}$, Rutten FFH, et al. Differences in overweight and obesity among children from migrant and native origin: a systematic review of the European literature. Obes Rev 2011;12:e535-47.

25 Fazel M, Reed RV, Panter-Brick C, et al. Mental health of displaced and refugee children resettled in high-income countries: risk and protective factors. Lancet 2012;379:266-82.

26 Woodland L, Burgner D, Paxton G, et al. Health service delivery for newly arrived refugee children: a framework for good practice. $J$ Paediatr Child Health 2010;46:560-7.

27 Kadir A, Battersby A, Spencer N, et al. Children on the move in Europe: a narrative review of the evidence on the health risks, health needs and health policy for asylum seeking, refugee and undocumented children. BMJ Paediatr Open 2019;3:1-3. doi:10.1136/bmjpo-2018-000364

28 Thomas J, Brunton J, Graziosi S. EPPI-Reviewer 4: software for research synthesis. EPPI-Centre software. London: Social Science Research Unit, UCL Institute of Education, 2018.

29 Moher D, Liberati A, Tetzlaff J, et al. Preferred reporting items for systematic reviews and meta-analyses: the PRISMA statement. Int $J$ Surg 2010;8:336-41.

30 Higgins JP, Green S. Cochrane handbook for systematic reviews of interventions: John Wiley \& Sons 2011.

31 DerSimonian R, Laird N. Meta-Analysis in clinical trials. Control Clin Trials 1986;7:177-88.

32 Campbell M, McKenzie JE, Sowden A, et al. Synthesis without meta-analysis (swim) in systematic reviews: reporting guideline. BMJ 2020;368:16890. 